\title{
Cable Design for FAIR SIS 300
}

J. Kaugerts, G. Moritz, M. N. Wilson, Member, IEEE, A. Ghosh, A. den Ouden, I. Bogdanov, S. Kozub, P. Shcherbakov, L. Shirshov, L. Tkachenko, D. Richter, A. Verweij, G. Willering, P. Fabbricatore, and G. Volpini

\begin{abstract}
GSI, Darmstadt is preparing to build FAIR (Facility for Antiproton and Ion Research) which will include SIS 300, a $300 \mathrm{~T} \cdot \mathrm{m}$ fast-ramping heavy ion synchrotron. Dipoles for this ring will be $2.9 \mathrm{~m}$ long, producing $6 \mathrm{~T}$ over a $100 \mathrm{~mm}$ coil aperture and ramped at $1 \mathrm{~T} / \mathrm{s}$. The cable for these dipoles must have low losses and produce acceptable field distortions during the fast ramp. We plan to achieve this objective by using fine $(\sim 3 \mu \mathrm{m})$ filaments of $\mathrm{NbTi}$ in a wire with an interfilamentary matrix of CuMn to reduce proximity coupling and increase the transverse resistivity. The Rutherford cable will have a thin stainless steel core and the wires will be coated with SnAg solder which has been oxidized, using a recipe similar to that developed at CERN, to increase the adjacent strand resistance Ra. Measurements of crossover strand resistance $R c$ and $R a$ in cored cable with oxidized SnAg coating will be presented, together with data on critical current, persistent current magnetization and eddy current coupling in a wire with ultra fine filaments and a CuMn matrix in the interfilamentary region of the wire. These data will be used to predict losses and field distortion in the SIS 300 dipole and optimize the final design of cable for FAIR.
\end{abstract}

Index Terms-AC loss, fast-ramping magnet, fine filament wire, inter-strand resistance, low loss cable.

\section{INTRODUCTION}

$\mathbf{U}$ NLIKE recently built colliding beam accelerators such as RHIC and now LHC, whose dipoles are ramped at rather modest ramp rates $(0.042 \mathrm{~T} / \mathrm{s}$ and $0.007 \mathrm{~T} / \mathrm{s}$, respectively) and remain at operating field for hours, the main dipoles for the FAIR SIS 300 synchrotron will be ramped between 1.6 and $6 \mathrm{~T}$, at $1 \mathrm{~T} / \mathrm{s}$, with the ramping time constituting $50 \%$ of the duty cycle. Minimization of conductor AC losses during such an operating mode is therefore required, to keep refrigeration costs

Manuscript received August 25, 2006.

J. Kaugerts and G. Moritz are with the Gesellschaft für Schwerionenforschung $\mathrm{mbH}$ (GSI), D-64291 Darmstadt, Germany (e-mail: J.Kaugerts@gsi.de; G:Moritz@gsi.de).

M. N. Wilson is a consultant at 33 Lower Radley, Abingdon OX14 3AY, U.K. (e-mail: m-wilson@dsl.pipex.com).

A. Ghosh is with the Brookhaven National Laboratory, Upton, NY USA (e-mail: aghosh@bnl.gov).

A. den Ouden is with the University of Twente, Enschede, The Netherlands (e-mail: a.denouden@utwente.nl).

I. Bogdanov, S. Kozub, P. Shcherbakov, L. Shirshov, and L. Tkachenko are with the Institute for High Energy Physics, Protvino, Russian Federation (e-mail: Igor.Bogdanov; Petr.Shcherbakov; Leonid.Shirshov; Leonid.Tkachenko@ihep.ru).

D. Richter, A. Verweij, and G. Willering are with the CERN, Geneva, Switzerland (e-mail: David.Richter@cern.ch; Arjan.Verweij@cern.ch; Gerard.Willering@cern.ch).

P. Fabbricatore is with the INFN, Genoa, Italy (e-mail: Pasquale.Fabbricatore@ge.infn.it).

G. Volpini is with the INFN, Milan, Italy (e-mail: Giovanni.Volpini@ mi.infn. it).

Color versions of one or more of the figures in this paper are available online at http://ieeexplore.ieee.org.

Digital Object Identifier 10.1109/TASC.2007.898474
TABLE I

SIS 300 CONDUCTOR DESIGN PARAMETERS

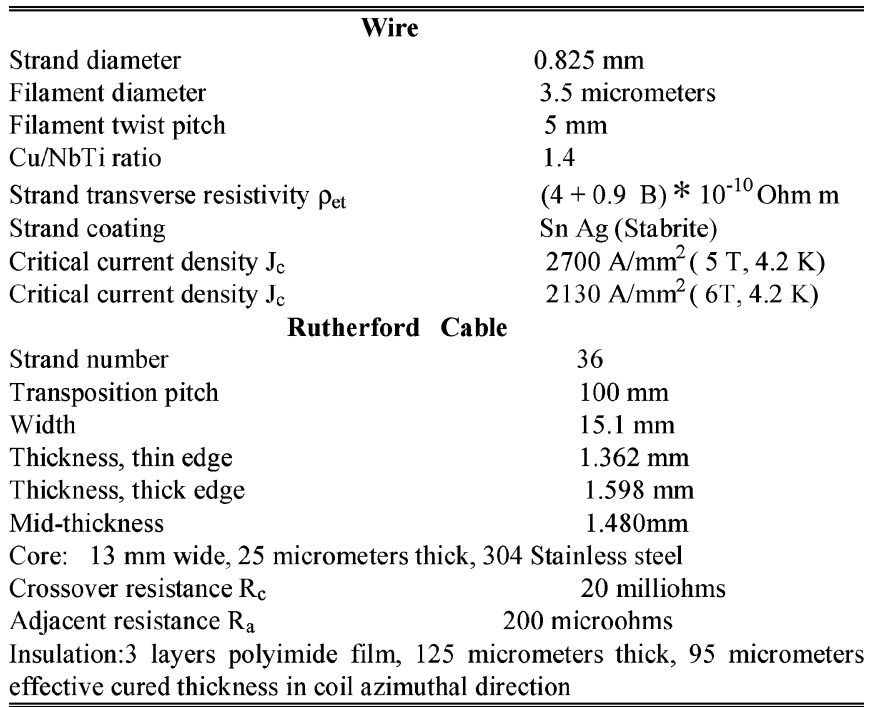

and conductor temperature margin at acceptable levels. The SIS 300 magnets will operate in supercritical helium, with an inlet temperature of $4.4 \mathrm{~K}$ and peak magnet temperature of $4.76 \mathrm{~K}$.

The development of a low loss conductor began when it was planned to build the SIS 200 synchrotron [1] with 4 T dipoles. The project requirements were changed, to the present SIS 300 synchrotron, with $6 \mathrm{~T}$ dipoles. A Rutherford cable with a central stainless steel core was adopted as the conductor for this design, as well as for the SIS 300 dipole design, although the strand size and number were both increased for the 6 T SIS 300 dipole. The details of such a cable and equations for different types of losses are given in [1]. The conceptual SIS 300 dipole design with design parameters was presented at ASC 2004 [2]. The final magnetic and mechanical design has been completed and will be presented [3]. The SIS 300 cable design parameters from ASC 2004 are presented in Table I.

The calculated conductor loss/cycle per meter of dipole magnet [4], using these parameters, is given in Table II. Hysteresis losses in the iron yoke add $26 \mathrm{~J} / \mathrm{m}$ to the cycle loss.

These conductor losses depend on the values of the cable crossover resistance $R_{c}$ and adjacent strand resistance $R_{a}, N b T i$ filament diameter $\mathrm{d}_{\mathrm{f}}$, and strand transverse resistivity $\rho_{\text {et }}$, as well as cable geometry, filament twist pitch, and strand transposition pitch. The values of these parameters given in Table I were chosen as achievable goal values, based on past experience. The cable dimensions were chosen to be the same as those of the LHC dipole outer layer cable. The progress in achieving these values as well as possible changes of values, to reduce conductor AC cycling losses, will be described. 
TABLE II

LOSS PER CYCLE (Joules/m)

\begin{tabular}{ll}
\hline \hline Transverse $\mathrm{B}$, crossover $\mathrm{R}_{\mathrm{c}}$ & 3.2 \\
Transverse $\mathrm{B}$, adjacent $\mathrm{R}_{\mathrm{a}}$ & 10.7 \\
Parallel $\mathrm{B}$ adjacent $\mathrm{R}_{\mathrm{a}}$ & 0.1 \\
matrix coupling current & 15.0 \\
Filament hysteresis & 47.9 \\
\hline Total magnet & $\mathbf{7 6 . 9}$ \\
\hline
\end{tabular}

\section{LOW LOSS CONDUCTOR DEVELOPMENT}

\section{A. Filament Hysteresis Loss}

The filament hysteresis loss is proportional to the filament diameter and its critical current density. If the filament diameter is reduced in a strand with an all-copper matrix, the filament spacing (s) to filament diameter (d) ratio (s/d) is kept constant, to avoid filament distortion (sausaging) which decreases the conductor critical current density $\mathrm{J}_{\mathrm{c}}$ [5], [6]. As the filament separation decreases, proximity coupling [7] sets in, increasing the effective filament size. The limit for filament size in a strand with an all-copper matrix is therefore limited to about $3.5 \mu \mathrm{m}$, before the onset of proximity coupling. The use of a $\mathrm{Cu}-0.5 \mathrm{wt} \% \mathrm{Mn}$ interfilamentary matrix can suppress proximity coupling down to filament sizes around $1 \mu \mathrm{m}$. $\mathrm{Cu}-0.5 \mathrm{wt} \% \mathrm{Mn}$ is also more resistive than copper at NbTi-based superconducting magnet operating temperatures ( 4-6 K), so that its use as the interfilamentary matrix material for a strand allows a reduction of filament diameter and thus hysteresis loss as well as coupling current losses, compared to a strand with an all-copper matrix. $\mathrm{Cu}-15 \% \mathrm{Ni}$ could also be used. However, it is more resistive than $\mathrm{CuMn}$, has a lower thermal thermal conductivity, and is also harder. Hence, $\mathrm{Cu}-0.5 \mathrm{wt} \% \mathrm{Mn}$ interfilamentary matrix wire (with copper outer jacket and inner core) appears as the best choice to reduce hysteresis and coupling current losses.

There was interest in the use of a strand with a CuMn interfilamentary matrix and $2.5 \mu \mathrm{m}$ filaments in the Superconducting Super Collider (SSC) days, for use in the SSC's High Energy Booster (HEB), which was ramped to $6.67 \mathrm{~T}$ at $0.07 \mathrm{~T} / \mathrm{s}$. A number of full sized billets of such wire were made, using both single and double stacking techniques. The concerns were (and still are) critical current density value, filament distortion (leading to a larger effective filament diameter, in terms of AC losses), and long enough strand piece length. A single stacking approach leads to less filament distortion, but requires starting elements that are too small to handle. Some still existing SSC HEB dipole outer layer $0.648 \mathrm{~mm}$ diameter wire [8] with $2.6 \mu \mathrm{m}$ filaments, made by a triple extrusion, double stacking process, was found. This conductor was tested at Twente TU (time and magnetic flux density $\mathrm{B}$ dependent magnetization measurements) and BNL (critical current density). The transport critical current density $\mathrm{J}_{\mathrm{ct}}$ (at $5 \mathrm{~T}, 4.2 \mathrm{~K}$ ) was $2511 \mathrm{~A} / \mathrm{mm}^{2}$, while the critical current density $\mathrm{J}_{\mathrm{cm}}$ calculated from the measured magnetization, assuming round filaments, was $3088 \mathrm{~A} / \mathrm{mm}^{2}$, giving a $\mathrm{J}_{\mathrm{cm}} / \mathrm{J}_{\mathrm{ct}}$ ratio of 1.23 . This can be interpreted as an effective filament distortion, reducing $\mathrm{J}_{\mathrm{ct}}$, or as an increase of the effective filament diameter, increasing hysteresis loss, as Fig. 1 shows for this only wire (B944-2) with CuMn interfilamentary matrix, as well as for the other all-copper matrix tested wires. The larger ratio of $\mathrm{J}_{\mathrm{cm}} / \mathrm{J}_{\mathrm{ct}}$ can also be associated with
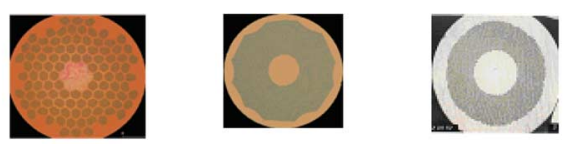

Strand dia

$2 \mathrm{~A} 2120.53 \mathrm{~mm}$

3N7 $0.42 \mathrm{~mm} \quad$ RHIC $\quad 0.64 \mathrm{~mm}$

RRR

$\rho_{\mathrm{et}}\left(10^{-10} \Omega \cdot \mathrm{m}\right)$

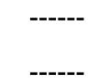

$----$

198

$\mathrm{J}_{\mathrm{c}}\left(5 \mathrm{~T}, 4.2 \mathrm{~K}, \mathrm{~A} / \mathrm{mm}^{2}\right) 2558$

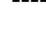

$(1.24+\quad 0.9 \mathrm{~B})$

filament dia.

$(\mu \mathrm{m})$

3.4

2831

3029

Ratio $\mathrm{J}_{c m} / J_{c t}$

1.40

3.4

5.71

0.94

0.92
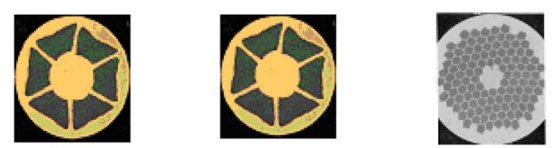

Strand dia. K201T4 0.67mm G201T6 $0.85 \mathrm{~mm}$ B944-2 $0.65 \mathrm{~mm}$

RRR

173

166

102

$\rho_{\mathrm{et}}\left(10^{-10} \Omega \cdot \mathrm{m}\right)(0.58+0.89 \mathrm{~B})$

$(0.82+0.46 \mathrm{~B})$

$(4.15+$

$1.9 \mathrm{~B})$

$\mathrm{J}_{\mathrm{c}}\left(5 \mathrm{~T}, 4.2 \mathrm{~K}, \mathrm{~A} / \mathrm{mm}^{2}\right) 2773$

2773

2511

filament dia.

$(\mu \mathrm{m}$

3.4

4. 3

2.6

Ratio $J_{c m} / J_{c t}$

1.15

1.10

1.23

Fig. 1. Characteristics of tested wires.

greater visible physical distortion of the filament cross sections, although the physical distortion of the filaments at the edge of the filament bundles is not as severe as one might expect, from the $\mathrm{J}_{\mathrm{cm}} / \mathrm{J}_{\mathrm{ct}}$ ratio. In general, the single stacked wires of Fig. 1 (3N7 \& RHIC) have a higher $\mathrm{J}_{\mathrm{ct}}$ value than the other (double stacked) wires. The attempt to reduce filament distortion by reducing the number of filament bundles (K201T4 \& G201T6), reduced $\rho_{\text {et }}$.

The $\mathrm{J}_{\mathrm{ct}}$ value given in [6] for the SSC HEB wire is about $2760 \mathrm{~A} / \mathrm{mm}^{2}(5 \mathrm{~T}, 4.2 \mathrm{~K}$ ) but this was for the best samples, whereas our measured sample $\left(\mathrm{J}_{\mathrm{ct}}=2511 \mathrm{~A} / \mathrm{mm}^{2}\right)$ was from a lot of $130 \mathrm{~kg}$ of remaining wire, with an average piece length of $1 \mathrm{~km}$. Commercially available $0.57 \mathrm{~mm}$ wire (strand) with a CuMn interfilamentary matrix and about $5 \mu \mathrm{m}$ filaments has been made by Outokumpu (now called Luvata), with a single stacking process (cross section like RHIC wire of Fig. 1), giving $\mathrm{J}_{\mathrm{ct}}$ values above $3000 \mathrm{~A} / \mathrm{mm}^{2}$. GSI is working together with INFN and industry to develop such a wire for the SIS 300 dipole, with the goal of an effective filament size of $2-3 \mu \mathrm{m}$ and a $\mathrm{J}_{\mathrm{ct}}$ value around $2700 \mathrm{~A} / \mathrm{mm}^{2}$. Due to filament size, this will require a double stacking approach and most likely result in some $\mathrm{J}_{\mathrm{c}}$ degradation, compared to the single stacked wire.

\section{B. Matrix Coupling Current Loss}

This is an eddy current loss, due to coupling currents induced in a current loop that includes the superconducting NbTi filaments as well as the strand matrix, plus normal eddy currents that circulate only in the matrix material. The problem has been treated by Turck, Duchateau and Ciazynski [9], [10].

The transverse resistivity $\rho_{\text {et }}$ for these coupling currents is given by

$$
\rho_{e t}=\frac{\dot{B}}{M}\left(\frac{p}{2 \pi}\right)^{2}
$$


where $\dot{B}$ is the time rate change of magnetic flux density $\mathrm{B}, \mathrm{M}$ is the magnetization, and $\mathrm{p}$ is the filament twist pitch.

Magnetization loops, taken at different values of $\mathrm{B}$ and $\dot{B}$ give the transverse resistivity as well as the magnetoresistance of a strand. Given that the SIS 300 cable must be heat treated to increase its $R_{a}$ value to the goal value given in Table I and that the magnet coils also undergo a $\sim 190^{\circ} \mathrm{C}$ cure cycle, to glue the turns of the polyimide tape insulated coil together, the RRR of a strand with an all-copper matrix will be high and the transverse resistivity can be expected to be similar to that of the RHIC wire (See Fig. 1). A wire with a CuMn interfilamentary matrix (whose resistivity at $12 \mathrm{~K}$ is $2.1 \bullet 10^{-8} \Omega \cdot \mathrm{m}$, versus $0.85 \bullet 10^{-10} \Omega \cdot \mathrm{m}$ for copper with $\mathrm{RRR}=200$ ) would give a higher value for the strand transverse resistivity and therefore a lower coupling current loss. The transverse resistivity of the SSC wire (B944-2 in Fig. 1) was measured to be $\rho_{\text {et }}=$ $(4.15+1.9 \mathrm{~B}) \bullet 10^{-10} \Omega \cdot \mathrm{m}$. The calculated value of $\rho_{\mathrm{et}}[11]$ for this wire is $4.70 \bullet 10^{-10} \Omega \cdot \mathrm{m}$, showing fairly good agreement between measurement and calculation for $\rho_{\text {et }}(\mathrm{B}=0)$. This is not true for the magnetoresistivity component of $\rho_{\text {et }}$ which must be determined experimentally.

However, this wire was measured, as received, without any heat treatment (which is needed to simulate a coil cure cycle or to increase cable $R_{a}$ ) and has a measured RRR value of 102 . The calculated $\rho_{\text {et }}$ value, for a RRR value of 200 for this wire, is $2.5 \bullet$ $10^{-10} \Omega \cdot \mathrm{m}$. Therefore, a more complicated strand design [12], with CuMn surrounding not only individual NbTi filaments, but also surrounding bundles of filaments encased in copper, will be required, to reach the goal value of $4 \bullet 10^{-10} \Omega-\mathrm{m}$ for $\rho_{\text {et }}$. Such a wire will also need a higher matrix/NbTi ratio (1.6:1), to accommodate enough copper for strand stability.

\section{CABLE $R_{c}$ AND $R_{a}$ LOSSES}

The $R_{c}$ and $R_{a}$ goal values of Table I were chosen as values that would reduce the $R_{c}$ and $R_{a}$ contributions to the total magnet loss to around $10 \%$ or less and as values that could be reasonably achieved.

The effectiveness of a central thin stainless steel core inside a Rutherford cable, in reducing the eddy current losses due to cable crossover resistance $R_{c}$, has already been shown [13]. In addition, the use of a core allows one to adjust the adjacent strand resistance $R_{a}$ independently of $R_{c}$. The $R_{c}$ goal value of $20 \mathrm{~m} \Omega$ can be easily reached with a $200^{\circ} \mathrm{C}$ heat treatment of several hours [14].

As for $\mathrm{R}_{\mathrm{a}}$, the conventional wisdom has been that one should make $R_{a}$ high enough to reduce cable eddy current losses to acceptable levels, but not so high that the cable's quench recovery ability through current sharing with adjacent strands of a quenching strand should be adversely affected. GSI has chosen to use Staybrite ( $\mathrm{Sn} 95 \mathrm{wtAg} 5 \mathrm{wt}$ ) solder, the same strand coating as chosen by CERN for the LHC main dipoles, as the strand coating for SIS 300 dipoles. Samples of LHC dipole outer layer cable, but with a 304 SS core, were first heat treated in air for a number of hours and then subjected to $R_{a}$ measurements [15]. Afterwards, they underwent two simulated coil cure cycles with an $R_{a}$ measurement after each cycle (see Fig. 2).

One can see that an approximately 6 hour heat treatment and one subsequent cure cycle is enough to get an $R_{a}$ value of $200 \mu \Omega$. However, the inner coil of the two layer SIS 300

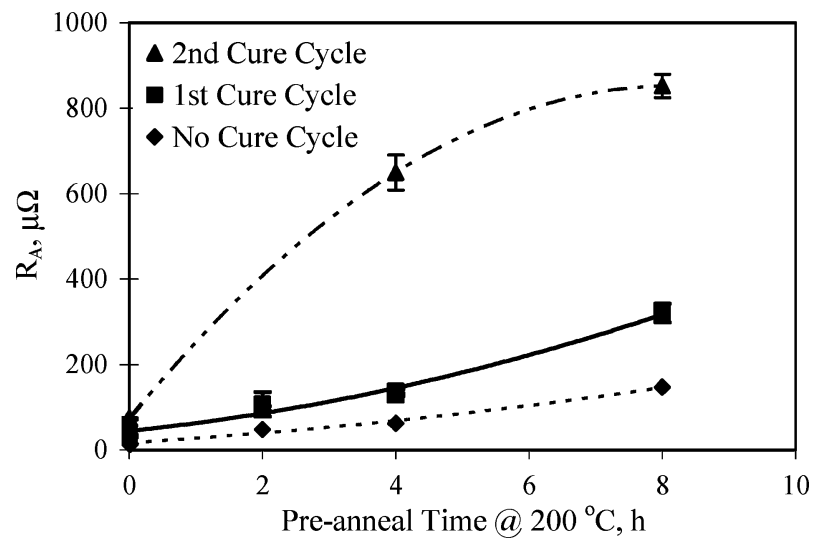

Fig. 2. $\mathrm{R}_{\mathrm{a}}$ for samples pre-annealed for different durations at $200^{\circ} \mathrm{C}$ in air (lower curve) and subsequently subjected to simulated coil cure cycles (upper 2 curves).

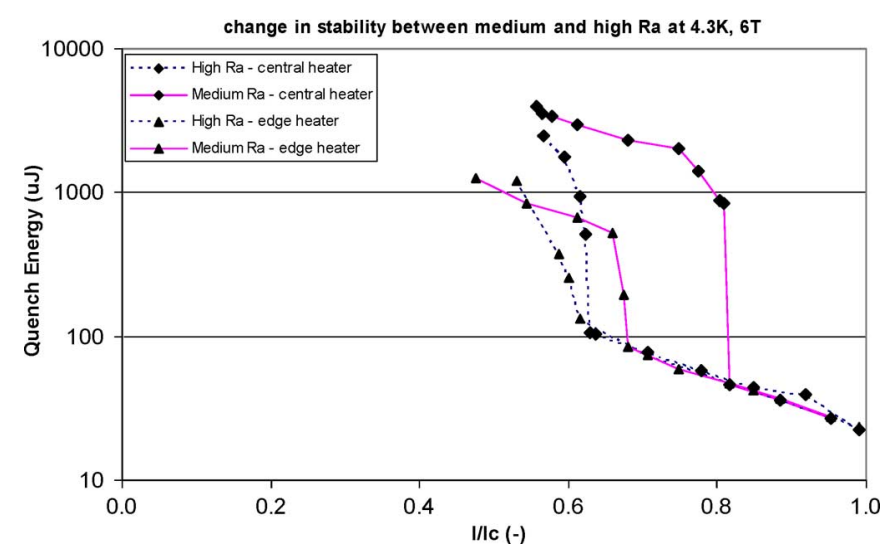

Fig. 3. Quench energy $(\mu \mathrm{J})$ versus fraction of critical current $\mathrm{I} / \mathrm{I}_{\mathrm{c}}$ for medium and high $R_{a}$ cable samples at $4.3 \mathrm{~K}, 6 \mathrm{~T}$.

dipole will be subjected to two cure cycles since it will be built without an interlayer splice. This means that the cable heat treatment time will need to be optimized to achieve the lowest cable $\mathrm{R}_{\mathrm{a}}$ loss for the magnet.

\section{CABle $R_{\mathrm{a}}$ EFfect on Minimum Quench Energy}

Minimum Quench Energy (MQE) measurements of Rutherford cables have been made before [16].

An MQE experiment (in liquid helium at $4.3 \mathrm{~K}$ ) was undertaken at CERN to measure the effect of cable $R_{a}$ value on the MQE of a cored LHC dipole outer layer cable [17], to determine what the maximum value of $R_{a}$ could be, before cable stability is affected. Low $(\sim 1 \mu \Omega)$, medium $(0.6-0.7 \mathrm{~m} \Omega)$, and high $\left(8-9 \mathrm{~m} \Omega\right.$ ) $\mathrm{R}_{\mathrm{a}}$ samples (using CERN arbitrary nomenclature for the $R_{a}$ values) were prepared. Initial results are shown for the medium and high $R_{a}$ samples (see Fig. 3), for $100 \mu$ sec pulses from a heater in the center of the cable and near the thin edge of the cable.

One can see that the "knee" of the curve (indicating the onset of current sharing of the quenching strand with adjacent strands) is at about $\mathrm{I} / \mathrm{I}_{\mathrm{c}}=0.81$ for the medium $\mathrm{R}_{\mathrm{a}}$ sample, but only 0.61 for the high $R_{a}$ sample. Therefore, the medium $R_{a}$ cable does appear to be more stable, in terms of MQE. Whether this is an electrical effect $\left(R_{a}\right.$ value) or thermal effect (heat transfer value 
between strands) is not yet known. All one can say is that if one heat treats the cable such that the $R_{a}$ values reaches the above given values, the medium $R_{a}$ cable has a higher quench energy than the high $\mathrm{R}_{\mathrm{a}}$ cable. An estimation was made of $\mathrm{I} / \mathrm{I}_{\mathrm{c}}$ for the SIS 300 dipole at $6 \mathrm{~T}(6.42 \mathrm{~T}$ max field and maximum conductor temperature $4.76 \mathrm{~K}$ ), assuming the cable parameters of Table I and uniform distribution of transport current among the strands, with the result $\mathrm{I} / \mathrm{I}_{\mathrm{c}}=0.75$.

\section{CONCLUSION}

The progress in achieving fine filament, low loss Rutherford cable superconductor for SIS 300 ring dipoles has been described. Goal values for filament diameter $\mathrm{d}_{\mathrm{f}}$, transverse resistivity $\rho_{\text {et }}$, cable resistances $R_{a}$ and $R_{c}$ have either been achieved in the past, or look achievable, based on tests and calculations. The critical current density $\mathrm{J}_{\mathrm{c}}$ goal in long lengths of fine filament strands with CuMn interfilamentary matrix still needs to be demonstrated. Initial test results show that conductor stability (MQE) is decreased as $R_{a}$ is increased.

\section{REFERENCES}

[1] M. N. Wilson, A. K. Ghosh, B. ten Haken, W. V. Hassenzahl, J. Kaugerts, G. Moritz, C. Muehle, A. den Ouden, R. Soika, P. Wanderer, and W. A. J. Wessel, "Cored Rutherford cable for the GSI fast ramping synchrotron," IEEE Trans. Applied Superconduct., vol. 13, no. 2, pp. 1704-1709, 2003.

[2] J. E. Kaugerts, G. Moritz, C. Muehle, A. Ageev, I. Bogdanov, S. Kozub, P. Shcherbakov, V. Sytnik, L. Tkachenko, V. Zubko, D. Tommasini, M. N. Wilson, and W. Hassenzahl, "Design of a $6 \mathrm{~T}$, $1 \mathrm{~T} / \mathrm{s}$ fast-ramping synchrotron magnet for GSI's planned SIS 300 accelerator," IEEE Trans. Applied Superconduct., vol. 15, no. 2, pp. $1225-1227,2005$.
[3] S. Kozub, L. Tkachenko, V. Zubko, E. Floch, J. Kaugerts, G. Moritz, B. Auchmann, S. Russenschuck, and D. Tommasini, "Magnetic and thermal characteristics of a model dipole magnet fort he SIS 300," in These Proceedings.

[4] M. N. Wilson, "Calculation of Loss in the SIS 300 Dipole," Report GSI 20 version 1, July 22, 2004, (GSI Internal Report).

[5] E. Gregory, T. S. Kreilick, J. Wong, A. K. Ghosh, and W. B. Sampson, "Importance of spacing in the development of high current densities in multifilamentary superconductors," Cryogenics, vol. 27, pp. 178-182, 1987.

[6] E. Gregory, "Recent developments in multifilamentary Nb-Ti superconductors," Cryogenics, vol. 27, pp. 290-297, 1987.

[7] A. K. Ghosh, W. B. Sampson, and Miller, "The effect of magnetic impurities and barriers on the magnetization and critical current of fine filament NbTi composites," IEEE Trans. Magnetics, vol. 24, no. 2, pp. $1145-1148,1988$.

[8] H. C. Kanithi, P. Valaris, and B. A. Zeitlin, "Superconductors with 2.5 micron NbTi filaments," in Supercollider 3, J. Nonte, Ed. New York: Plenum Press, 1991.

[9] B. Turck, "Coupling losses in various outer normal layers surrounding the filament bundle of a superconducting composite," J. Appl Phys., vol. 50, no. 8, pp. 5397-5401, 1979.

[10] J. L. Duchateau, B. Turck, and D. Ciazynski, "Coupling-current losses in composites and cables: Aanalytical calculations," in Handbook of Applied Superconductivity, B. Seeber, Ed. London: Institute of Physics, vol. 1, ch. B4.3.

[11] M. N. Wilson, "Magnetization of SSC CuMn Wire Type B944-2," Report GSI 25 version 5, August 10, 2006, (GSI Internal Report).

[12] M. N. Wilson, "Some Thoughts on Wire Design for SIS 300," Report GSI 29 version 1, January 10, 2006, (GSI Internal Report).

[13] M. N. Wilson, "AC Losses and Temperature Rise in RHIC Dipole Magnets," Report GSI 1 version 3, December 31, 2000, (GSI Internal Report).

[14] I. Bogdanov, S. Kozub, V. Pokrovsky, P. Shcherbakov, L. Shirshov, L. Tkachenko, and V. Zubko, "Study of Current Carrying Element for SIS 300 Dipole," July 2005, IHEP Internal Report.

[15] A. K. Ghosh, "Inter-Strand Resistance of Prototype SIS 300 Dipole Cable," BNL Tech. Note 643-35, AM-MD-343.

[16] A. K. Ghosh, W. B. Sampson, and M. N. Wilson, "Minimum quench energies of rutherford cables and single wires," IEEE Trans. Applied Superconduct., vol. 7, no. 2, pp. 954-957, 1997.

[17] G. Willering, C. H. Denarie, S. Geminian, and A. Verweij, "New FRESCA Sample Holder for Cable Stability Experiments," CERN technical note 2005-11, EDMS 691690 (2005). 